\title{
Early-Design Optimization of Target Ventilation Rates for Hybrid Buildings Using Single-Node Analytical Model
}

\author{
Alpha Yacob Arsano ${ }^{1}$, Christoph Reinhart ${ }^{1}$, Carlos Cerezo ${ }^{1}$ \\ ${ }^{1}$ Massachusetts Institute of Technology, Cambridge, USA
}

\begin{abstract}
This manuscript presents an early-design methodology that identifies actionable design goals that maximize the effectiveness of natural ventilation in hybrid buildings. These design goals are presented using a compactness factor, recommended thermal mass area and target air change rates per hour ( $\mathrm{ACH}$ ) to maintain thermal comfort preferences of occupants. This approach will enable the consultant to explore viable design options that satisfy the identified design goals, such as optimum opening size based on the target $\mathrm{ACH}$ when wind and buoyancy ventilation forces are available, or incorporating fanassisted ventilation when natural ventilation is not sufficient. The method implements a single-node transient, analytical model using non-geometric mathematical representations of building parameters defined in a python routine.
\end{abstract}

\section{Introduction}

There is a growing recognition among practitioners and researchers for the importance of passive building strategies to meet environmental goals while improving occupants' comfort and productivity. Natural ventilation is an important passive building strategy, historically used to provide thermal comfort and maintain indoor air quality. The most critical design decisions determining the effectiveness of natural ventilation and building performance, such as massing, opening size and programmatic arrangement, are typically made in early design.

There have been many publications on natural ventilation in recent years, which presented various computational methodologies as well as experimental studies. The commonly used climate-file based methodologies play a crucial role in understanding the potential of a given climate at the beginning of a design project and provide preliminary suggestion on preferred passive building strategies. These climate file based methods are great starting points by evaluating outdoor conditions such as ambient temperature and humidity; however, they do not account for building parameters - envelope performance, thermal mass and internal loads - that greatly influence indoor conditions.

Other early-design methodologies that implement numerical thermal simulations play a great role in the evaluation of building strategies; however, their application during the schematic design stage is limited due to the complexity and the level of geometrical information required when setting up the thermal model. One example of such numerical simulation engines that is often used to enhance the design decision process is EnergyPlus, developed by the U.S. Department of Energy (DOE). The engine outputs results on indoor temperature, indoor humidity levels and energy loads by implementing a heat balance equation on a zone air (EnergyPlus, 2016). A previous approach that implemented the EnergyPlus engine to estimate hourly ventilation rates is the study by (Yoon \& Malkawi, 2017). They used simulated cooling demand of a fully conditioned building and calculated the equivalent number of $\mathrm{ACH}$ that can achieve the predicted cooling demand; their analytical model implemented conservation of energy equation based on simulated indoor temperature and cooling load. The limitation of this approach at an early-design stage is that the user is required to set up an energy model using a building information modelling (BIM) environment of Rhinoceros and an energy simulation script in a Grasshopper plugin called Honeybee.

Additionally, a growing body of literature is on model predictive control (MPC) of naturally ventilated and hybrid buildings. (Hu \& Karava, 2014), (May-Ostendorp, Henze, Corbin, Rajagopalan, \& Felsmann, 2011), (Spindler \& Norford, 2009a) and (Chen, Norford, Samuelson, \& Malkawi, 2018) have presented methodologies to control different modes of operation in hybrid buildings to maximize performance and reduce energy use. However, the use of MPC approach has not yet been explored as part of an early-design framework to guide designer's decisions on building strategies that maximize natural ventilation potential and reduce the use of active cooling.

The proposed approach hence presents an early-design decision support methodology that implements quick analytical calculations and the principles of MPC in 24hour cycles to recommend design goals for naturally ventilated and hybrid buildings. The required user inputs at the beginning of the analysis are only the location or climate of the project and the intended building program. Users have the option to specify comfort requirements by adjusting acceptable indoor temperature and humidity levels, as well as acceptable number of discomfort hours diurnally or annually. The methodology integrates the evaluation of outdoor conditions using climate data of a project's location and predicted indoor conditions for the intended building program using single-node analytical 
model. Finally, design goals are drawn in terms of compactness factor of a building's massing - the ratio of total floor area to the exposed envelope surface area, recommended thermal mass area and hourly ventilation rate given as target $\mathrm{ACH}$. The suggested design goals are intended to maximize the potential of natural ventilation and provide a pathway for their implementation in architectural design phase.

\section{Methodology}

The approach proposed in this document integrates the evaluation of outdoor conditions using climate data of a project's location and predicted indoor conditions for the intended building program. Building parameters are represented in a non-geometric analytical computation using a python script to calculate the potential of natural ventilation.

The recommended design goals are identified by comparing multiple combinations of design variables. The results discussed in this manuscript are based on over 500 different combinations and demonstrate that a broader range of possibilities can be explored to maximize natural ventilation effectiveness.

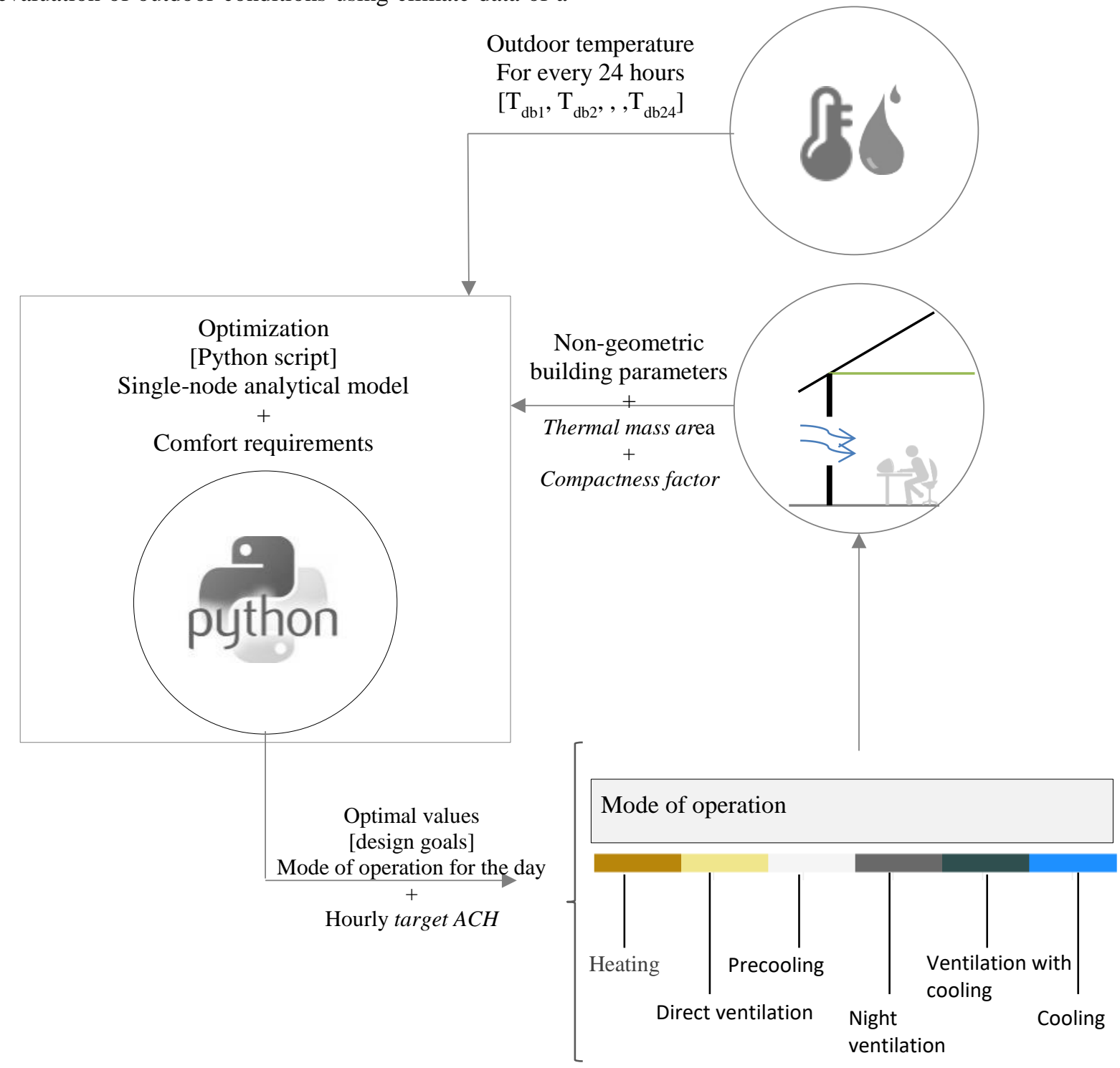

Figure 1: The proposed framework to evaluate different combinations of design variants and draw actionable design recommendations that maximize the effectiveness of natural ventilation in hybrid buildings. 


\section{Design constraints}

Climate: outdoor temperature and humidity

As a first step of the methodology, hourly outdoor temperature and relative humidity values are evaluated to identify if natural ventilation is possible based on userpreferred comfort evaluation criteria. The data on outdoor conditions are extracted from EnergyPlus weather data files (EWP) that are publicly available for over 7000 climates globally (US-DOE \& NREL, 2018).

The figure below shows an example of outdoor condition analysis with an acceptable comfort range given in strict set points for a project in Boston. The total number of hours that will meet both outdoor temperature (between 18 and $25^{\circ} \mathrm{C}$ ) and humidity (less than $70 \%$ ) requirements are 974 hours.

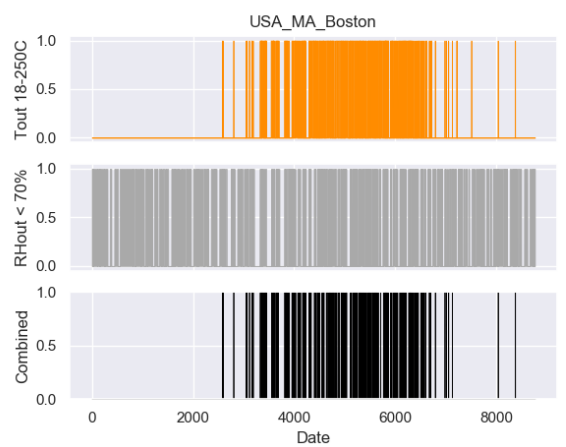

Figure 2: Outdoor temperature and humidity that are within a strict comfort requirement.

It is important to recognize that this first approach only assists to characterize a climate as hot-to-warm, temperate or cold, rather than providing complete information on the availability of natural ventilation. With the application of different complementary passive building strategies, such as thermal mass and night ventilation, the potential of natural ventilation can be significantly increased. Detailed discussion on different building parameters and modes of operation is presented in the following sections.

\section{Building program and parameters}

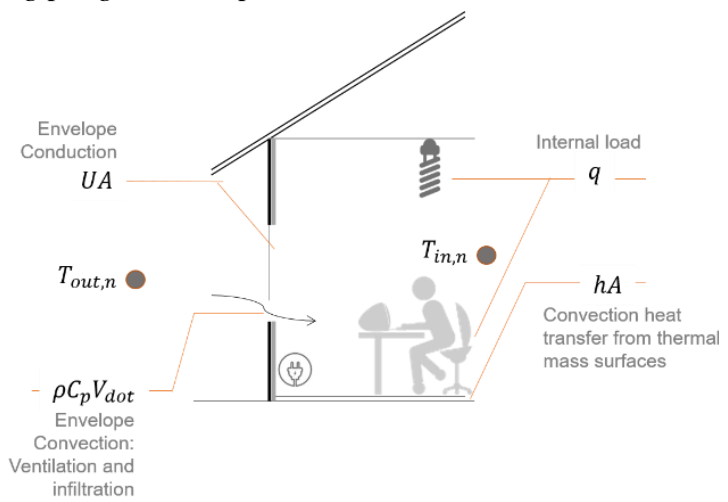

Figure 3: Mathematical expressions representing different building parameters. These expressions are integrated into an analytical model given in equations

$$
(1,2,3) \text {. }
$$

As shown in Figure 3, building parameters such as envelope performance, internal loads from lighting, equipment and occupants, and thermal mass, are represented mathematically in the analytical model as elaborated in the following sub-sections. The workflow can be used to study any building type including residential and commercial buildings. In this manuscript a medium-size office building is considered to illustrate the analysis. The standard ASHRAE 90.1 (ASHRAE, $2013 \mathrm{~b}$ ) is used as a reference to define occupancy schedules, lighting and equipment loads of a representative office building. Cooling degree days (CDD) and heating degree days (HDD) calculated using outdoor temperature are used to determine the climate zone of the selected climate based the information provided in ASHRAE 169 (Crawley et al., 2013).

Table 1: ASHRAE prescribed performances for a wall that is above grade and operable opening with metal framing for nonresidential building. $U$-values are given in $W / m 2 K$.

\begin{tabular}{cccc} 
& $\begin{array}{c}\text { Light } \\
\text { construction }\end{array}$ & $\begin{array}{c}\text { Mass } \\
\text { construction }\end{array}$ & $\begin{array}{c}\text { Vertical } \\
\text { Opening }\end{array}$ \\
\hline CZ0 & U-0.504 & U-3.293 & U-3.69 \\
\hline CZ1 & U-0.504 & U-3.293 & U-3.69 \\
\hline CZ2 & U-0.504 & U-0.857 & U-3.69 \\
\hline CZ3 & U-0.504 & U-0.701 & U-3.41 \\
\hline CZ4 & U-0.365 & U-0.592 & U-2.61 \\
\hline CZ5 & U-0.291 & U-0.513 & U-2.61 \\
\hline CZ6 & U-0.291 & U-0.453 & U-2.56 \\
\hline CZ7 & U-0.291 & U-0.404 & U-2.27 \\
\hline CZ8 & U-0.182 & U-0.273 & U-1.99
\end{tabular}

\section{Design variables: used to define design goals}

Compactness factor to represent building typologies

A compactness factor represents the ratio of the total occupied floor area and exposed building surface area façade and roof. A comparison of different design typologies with similar total floor area using a compactness factor provides useful information about the massing of the project. A low compactness factor indicates that the building has relatively smaller building depth and a large exposed surface, while a high compactness factor indicates that the building has deep floor plans potentially limiting indoor airflow but significantly reducing heat conduction losses through building envelope.

Furthermore, the total area of exposed external surfaces that is one of the inputs in the single-node analytical model can be calculated from the compactness factor. It is an important parameter because conduction heat losses of the building to the external environment take place through these external surfaces.

To illustrate the application of the compactness factor three variants for a hypothetical medium-sized office building are shown in Figure 4 based on the U.S. DOE Commercial Reference Building Models of the National 
Building Stock database. Furthermore, the data in standard ASHRAE 209 (ASHRAE, 2018) provides representative parameters for various building types when the rough building form has not been prescribed. The medium office building that is analysed in this manuscript has a total floor area of $4982 \mathrm{~m}^{2}$ and a glazing fraction of 0.33 .

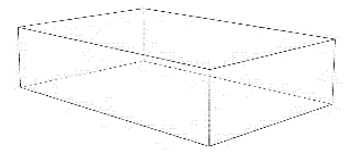

Afacade: $1972 \mathrm{~m}^{2}$

Aperfloor: $1660 \mathrm{~m}^{2}$ (3 floors)

Aroof: $1660 \mathrm{~m}^{2}$

Compactness factor: 1.38

Base building (medium office)

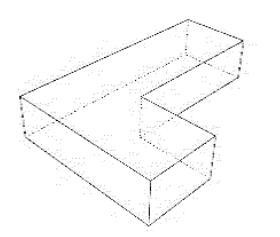

$\mathrm{A}_{\text {facade: }} 2448 \mathrm{~m}^{2}$

Aperfloor: $1650 \mathrm{~m}^{2}$ (3 floors)

Aroof: $1650 \mathrm{~m}^{2}$

Compactness factor: 1.20

Variant 1: with $12 \%$ lower compactness factor.

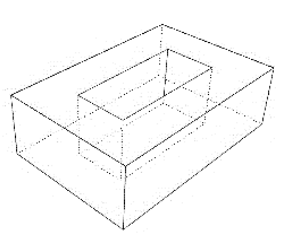

Afacade:5641 m²

Aperfloor: $1260 \mathrm{~m}^{2}$ (4 floors)

Aroof: $1260 \mathrm{~m}^{2}$

Compactness factor: 0.72

Variant 2: with $48 \%$ lower compactness factor.

Figure 4: Three massing prototypes representing medium office floor area based on DOE reference building.

\section{Thermal mass}

The thermal storage capacity of a building is recognized for improving indoor thermal comfort when ventilation is used to precool buildings at night or early hours of a day, and day-time ventilation is not allowed to prevent hot air from entering the building (Holford \& Woods, 2007). The thickness of the thermal mass that is actively involved in determining the thermal behaviour of a space is dependent on the thermal diffusivity of the thermal mass material and the diurnal temperature swing.

Table 2: Thermal mass parameters.

\begin{tabular}{|c|c|c|}
\hline $\mathbf{C ~ [ J / k g . K ]}$ & $\boldsymbol{\rho}(\mathbf{k g} / \mathbf{m} 3)$ & Thickness $(\mathbf{m})$ \\
\hline $\begin{array}{c}\text { Thermal mass } \\
\text { material } \\
\text { capacitance } \\
(1000)\end{array}$ & $\begin{array}{c}\text { Thermal mass } \\
\text { material density }\end{array}$ & $\begin{array}{c}\text { Thermal mass } \\
\text { thickness that is } \\
\text { thermally } \\
\text { activated (0.04) }\end{array}$ \\
\hline
\end{tabular}

The area of the thermal mass is taken as a design variant and is expressed in terms of a multiplication factor of the building's floor area. For the cases presented in the results section, a high thermal mass indicates exposed floors, ceilings and envelope construction - a factor of 2.5; a medium thermal mass indicates exposed floors or ceilings - a factor of one; a low thermal mass indicates thermal storage capacity of the envelope construction - a factor of 0.5 .

\section{Target air change rate}

To find the optimum target $\mathrm{ACH}$ for each hour of a day when natural ventilation is possible, the analytical equation in (3) is used - ACH values between 0.2 and 10, with an increment of $0.2 \mathrm{ACH}$, are compared for all thermal mass area scenarios that are discussed in the subsection above. In addition, three modes of natural ventilation are evaluated - day-time ventilation, night ventilation and precooling. Detailed descriptions are provided in the sub-section below modes of operation.

The optimization routine outputs the target $\mathrm{ACH}$ for the scenario where the lowest discomfort hours are achieved after comparing all combinations. Examples of target $\mathrm{ACH}$ charts are shown in the results section.

\section{Analytical model to calculate target $\mathrm{ACH}$ based on comfort temperature threshold}

The adopted single-node analytical model uses simplified mathematical expressions to predict hourly indoor temperature for a non-geometric abstraction of a building based on outdoor temperature, thermal resistances of the envelope and openings, internal loads, and the thermal response of the zone.

$$
\tau=R C
$$

The time-constant that represents the thermal response of the zone in a lumped variable $\tau$ is calculated as shown in (1) where: $R$ is the total resistance and $C$ is the thermal capacitance of the thermal mass as a product of mass $(m$ in $\mathrm{kg}$ ) and heat capacity of the material ( $c$ in $\mathrm{J} / \mathrm{kg} . K$ ). Figure 3 shows the various building parameters and their mathematical expressions.

The model is based on the principles of lumped parameter model (LPM) that is commonly used in various engineering fields such as electrical engineering and heat transfer, and it is a reliable approximation for quick thermal simulation (Spindler \& Norford, 2009b).

The conduction resistance of the zone's envelope and ventilation airflow, and convection resistance of the exposed thermal mass surface is given in (2). $U$ is the thermal transmittance of the building envelope in $\mathrm{W} / \mathrm{m}^{2} \mathrm{~K}$, $\rho$ is the density of air, $c_{p}$ is specific heat capacity of air, $V_{d o t}$ is the volumetric flowrate of air, $h$ is the convection coefficient inside the zone, and $A$ is the area of exposed thermal mass.

$$
R=\left(\frac{1}{\left(U A+\rho C_{p} V_{d o t}\right)}+\frac{1}{h A}\right)
$$

The hourly internal gains are calculated from lighting load, equipment load, occupants and solar gain through glazing. Table 3 shows an example based on the base DOE medium-office reference building. However, these values can be adjusted based on the performance goals of the project.

Table 3: Sources of internal loads.

\begin{tabular}{|c|c|c|}
\hline Lighting & Equipment & People \\
\hline $12 \mathrm{~W} / \mathrm{m}^{2}$ & $12 \mathrm{~W} / \mathrm{m}^{2}$ & $0.2 \mathrm{p} / \mathrm{m}^{2}$ \\
\hline
\end{tabular}


And finally (3) shows the overall analytical equation that is implemented to run over 500 combinations and recommend design goals for the case being analysed.

$$
\begin{aligned}
& \boldsymbol{T}_{\text {in }, \boldsymbol{n}+\mathbf{1}}=\left(\left(\frac{1}{\left(U A+\rho C_{p} V_{\text {dot }}\right)}+\frac{1}{h A}\right) q+T_{\text {out }, n}\right)(1- \\
& \left.e^{\frac{-1}{\tau}}\right)+\left(T_{i n, n} * e^{\frac{-1}{\tau}}\right)
\end{aligned}
$$

Where:

$T_{i n, n+l}$ is the indoor temperature at the next time step,

$T_{i n, n}$ is the indoor temperature at the current time step,

$T_{\text {out }, n}$ is the outdoor temperature at the current time step,

$q$ is total internal heat gain at each time step, and $\rho C_{p} V_{d o t}$ is the ventilation flowrate at each time step.

\section{Modes of operation}

Six different modes of operations are defined based on the passive and active strategies that can be implemented to meet the specified comfort requirements. The first mode of operation is heating - when the mean outdoor temperature is below $10{ }^{\circ} \mathrm{C}$ or when indoor temperature during occupied hours is less than $18^{\circ} \mathrm{C}$.
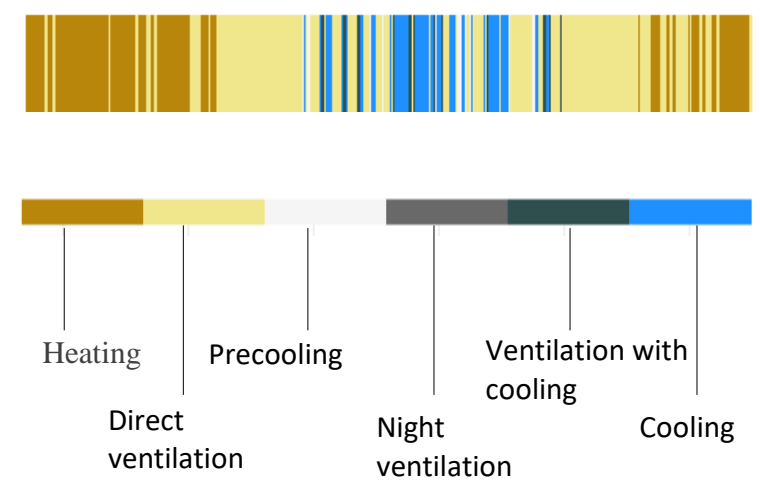

Figure 5: Different modes of operations for a medium office in Boston, assigned for each day of the year based on the lowest number of discomfort hours.

Three modes of operations are defined for natural ventilation. Direct ventilation mode is applicable when natural ventilation for cooling is possible during the occupied hours of the day - when outdoor air temperature is below the upper indoor comfort-temperature threshold. When outdoor temperatures are high, direct ventilation is not possible; hence, precooling mode and night ventilation modes are recommended. In the case of precooling, natural ventilation is used to precool the space during early hours between 6 am and 8 am, where outdoor temperature is relatively lower, while in the case of night ventilation precooling with natural ventilation is achieved during night hours between $10 \mathrm{pm}$ and $4 \mathrm{am}$. In these precooling strategies, it is assumed that there will not be day-time natural ventilation. In the cases presented in the results section, these three natural ventilation modes are applicable only when the number of diurnal discomfort hours are less than three.
The fourth mode of operation combines night ventilation and precooling; with active cooling only for limited number of hours - when the diurnal discomfort hours are between three and five hours. However, when the number of discomfort hours during the day are above five hours, active cooling will be on with no natural ventilation.

\section{Comfort evaluation}

The preference for indoor comfort conditions highly depends on the programmatic use of the building as well as occupants' preference. It is customary to use specific temperature and humidity set points when designing for more controlled environment such as office and commercial spaces. The adaptive comfort model in ASHRAE 55 can implemented when active cooling systems are not operated (ASHRAE, 2013a) and when there is acceptance for higher temperature ranges.

\section{Results}

This section presents the implementation of the proposed approach in three different climates that have different natural ventilation potential: Boston, Phoenix and San Francisco.

\section{Climate: availability of natural ventilation}

Boston, San Francisco and Phoenix are selected to demonstrate the proposed methodology. The comparison between these three climates showcases heating dominated, temperate and cooling dominated climates that have considerable potential for natural ventilation in different times of the year. Figure 6 presents availability of natural ventilation based on the comfort evaluation criteria discussed in the comfort evaluation sub-section above.

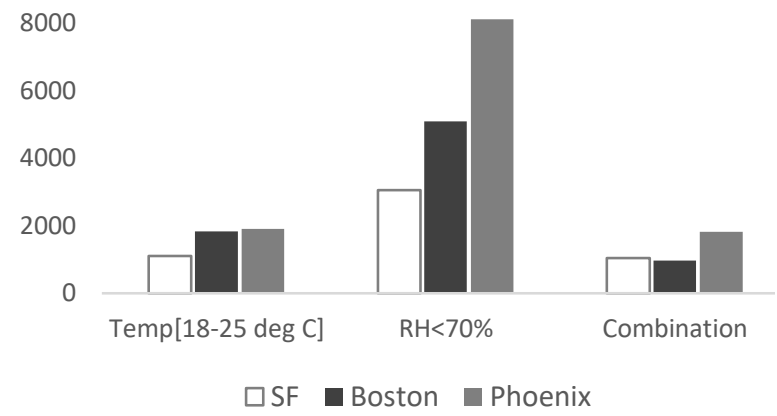

Figure 6: Number of hours representing outdoor temperature and humidity that are within comfort range, based on a strict comfort requirement in office building.

\section{Predicted target ACH for the best combinations}

The following figures show the predicted target $\mathrm{ACH}$ for each hour of the day to maintain thermal comfort requirements that are specified at the beginning of the project. The temporal heatmaps indicate the recommended mode of operation for each day - heating, natural ventilation, limited cooling with natural ventilation, or cooling. During days when natural 
ventilation is possible to supply fresh air and provide cooling, the target ventilation rate is predicted as hourly target $\mathrm{ACH}$.

For the examples illustrated in this section, the acceptable comfort threshold during warm or hot seasons is specified with indoor temperature upper limit of $25^{\circ} \mathrm{C}$. Discomfort hours due to high temperature are calculated for each day to compare different cases specified with different $\mathrm{ACH}$ values and exposed thermal mass area as illustrated in Figure 1.

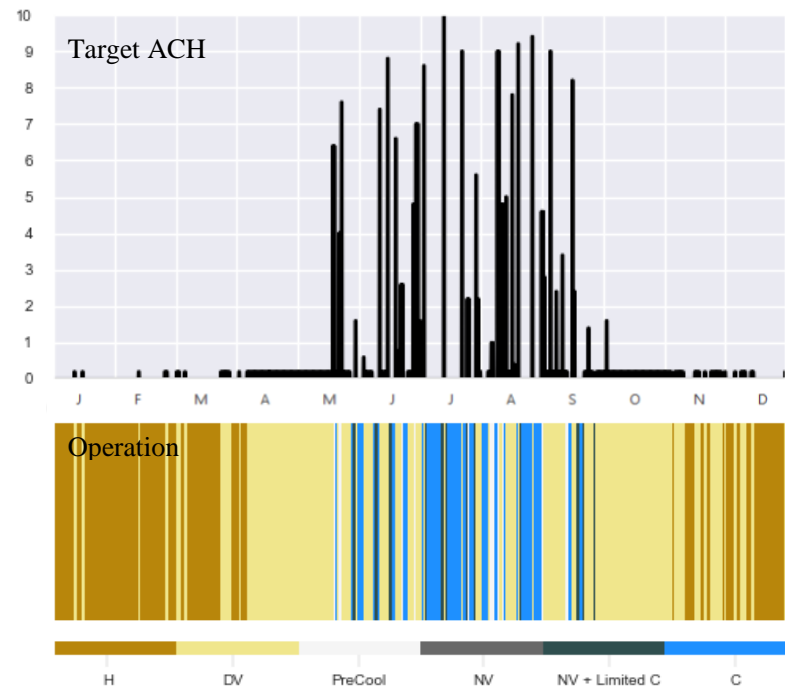

Figure 7: Boston: recommended target ACH values are shown in the upper chart. The lower chart shows when there is good natural ventilation potential and when active cooling should be to maintain comfort range during the summer season.
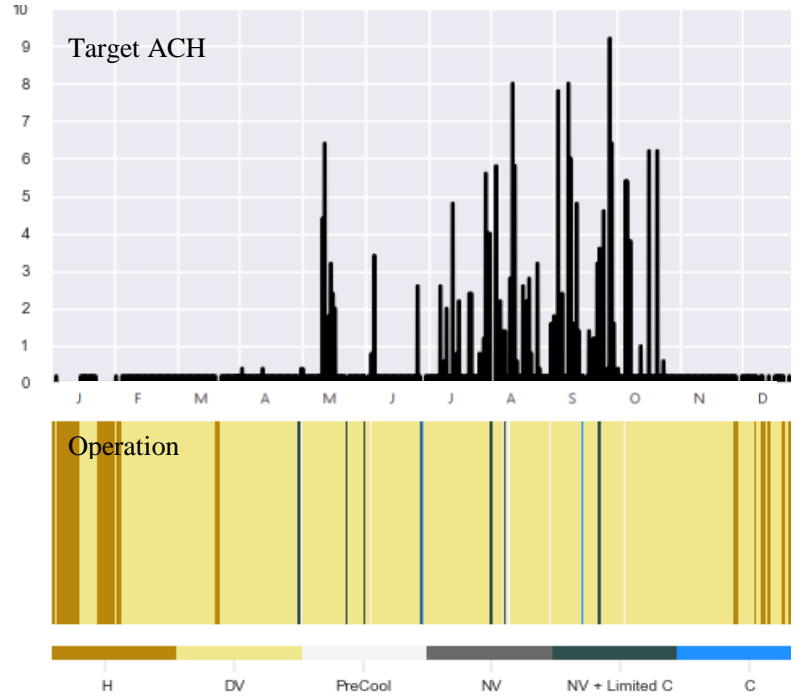

Figure 8: San Francisco: recommended target ACH values are shown in the upper chart. The lower chart shows that there is good natural ventilation potential and minimal active cooling can be used only few days in the year.
In the case of Boston, the high thermal mass, with exposed floor and ceiling area, is the preferred strategy - with total annual discomfort hours below 50 hours, it has the lowest number of days that require cooling ( 55 days) and the largest number of days with natural ventilation (188 days). See Figure 2 for thermal mass parameters. Recommended target $\mathrm{ACH}$ values go as high as 10 between May and August, but remain below 1 during the shoulder seasons.

In the case of San Francisco, medium thermal mass, with exposed floor or ceiling area, is the preferred strategy with total annual discomfort hours below 50 hours, it has the lowest number of days that require cooling (6 days) and the largest number of days with natural ventilation (316 days). Recommended target $\mathrm{ACH}$ values go as high as 9 in September, and remain below $5 \mathrm{ACH}$ during the rest of the year.

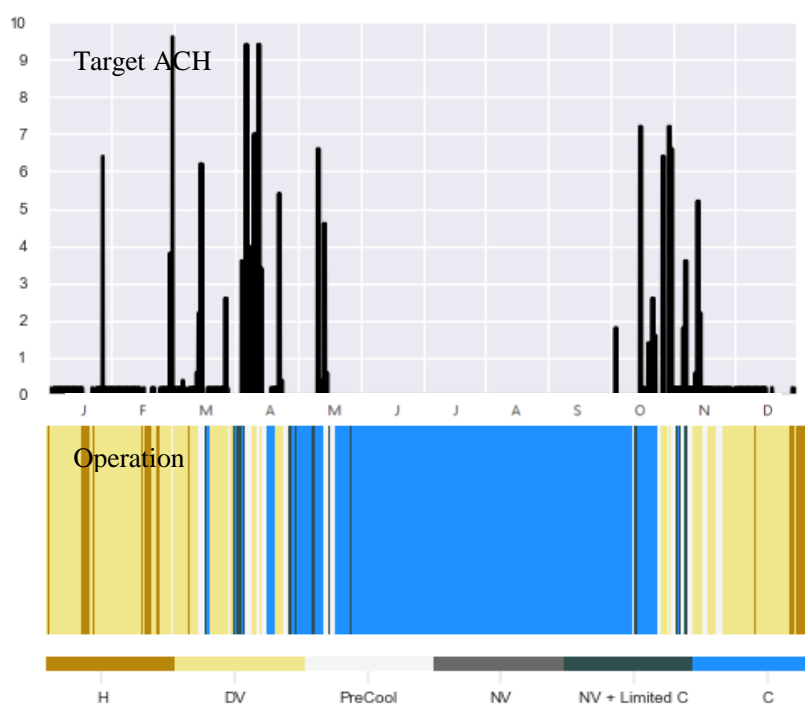

Figure 9: Phoenix: recommended target ACH values shown in the upper chart indicate that natural ventilation is possible for about $40 \%$ of the year. The lower chart shows that active cooling shall be considered between May and September when natural ventilation will not be sufficient to main the specified thermal comfort requirements.

In the case of Phoenix, very high thermal mass, with exposed floor and ceiling surfaces as well as mass envelope construction, is the preferred strategy - with total annual discomfort hours below 50 hours, it has the lowest number of days that require cooling (178 days) and the largest number of days with natural ventilation (153 days). See Figure 2 for thermal mass parameters. Recommended target $\mathrm{ACH}$ values go as high as 10 between February and April, and remain below $7 \mathrm{ACH}$ for the remaining months. 


\section{Conclusion and Discussion}

The goal of this paper is to bridge the gap between earlydesign, climate based natural ventilation evaluation methods and detailed building performance studies that are commonly implemented to calculate the effectiveness of natural ventilation. The methodology presented uses the underlining principle of MPC where the most suitable mode of operation - heating, cooling or natural ventilation - is recommended after evaluating multiple possibilities of building strategies for every 24 hours.

A transient analytical model for a single node is used to predict hourly indoor temperature for each day, and an optimization script based on thermal comfort requirements is implemented to compare various combinations of design variants - compactness factor, thermal mass area and target $\mathrm{ACH}$. This approach draws design goals that are actionable and promote the implementation of passive strategies in the design phase. The compactness factor can be used to inform decisions on massing of a building; target $\mathrm{ACH}$ in combination with other factors such as access to wind can be used to make decisions about floor layout, programmatic organization and airflow strategies. In the scenario where the hourly target $A C H$ are large and natural forces of ventilation such as wind and buoyancy are not available, fan-assisted natural ventilation can be considered.

Additionally, this manuscript presents results for three different climates to illustrate the proposed methodology. A heating dominated climate (Boston), a temperate climate (San Francisco) and a cooling dominated climate (Phoenix) have different natural ventilation potential. Non-geometric input parameters related to building program and total floor area are based on medium office U.S. DOE reference buildings. The findings of the study show that hybrid buildings in all climates would greatly benefit from natural ventilation: in Boston natural ventilation is possible $50 \%$ of the year, in San Francisco $85 \%$ of the year and in Phoenix $40 \%$ of the year.

To further enhance the proposed methodology, detailed exploration of objective optimization functions are being conducted. This ongoing work aims to enable the use of algorithms with multiple optimization criteria in addition to the lowest number of discomfort hours, including the lowest cooling energy and the minimum possible target $A C H$ values to comply with the project's comfort requirements. Furthermore, future work will showcase how various design goals can be successfully implemented in the design stage.

\section{References}

ASHRAE. (2013a). Standard 55-2013 - Thermal Environmental Conditions for Human Occupancy. ASHRAE. https://doi.org/ISSN 1041-2336
ASHRAE. (2013b). Standard 90.1-2013 Energy standard for buildings except low-rise residential buildings. Atlanta, GA: American Society of Heating, Refrigerating, and Air-Conditioning Engineers.

ASHRAE. (2018). ASHRAE 209-2018 Standard 209. 2018 -- Energy Simulation Aided Design for Buildings except Low-Rise Residential Buildings.

Chen, Y., Norford, L. K., Samuelson, H. W., \& Malkawi, A. (2018). Optimal control of HVAC and window systems for natural ventilation through reinforcement learning. Energy and Buildings. https://doi.org/10.1016/j.enbuild.2018.03.051

Crawley, D. B., Shirey, D. B., Cornick, S. M., Jarrett, P. L., Lott, J. N., Morris, R. J., ... Ferguson, J. M. (2013). Climatic data for building design standards. ASHRAE Standard, 8400(169).

EnergyPlus. EnergyPlus-Engineering Reference, US Department of Energy $\S \quad$ (2016). https://doi.org/citeulike-article-id:10579266

Holford, J. M., \& Woods, A. W. (2007). On the thermal buffering of naturally ventilated buildings through internal thermal mass. Journal of Fluid Mechanics. https://doi.org/10.1017/S0022112007005320

Hu, J., \& Karava, P. (2014). Model predictive control strategies for buildings with mixed-mode cooling. Building and Environment. https://doi.org/10.1016/j.buildenv.2013.09.005

May-Ostendorp, P., Henze, G. P., Corbin, C. D., Rajagopalan, B., \& Felsmann, C. (2011). Modelpredictive control of mixed-mode buildings with rule extraction. Building and Environment. https://doi.org/10.1016/j.buildenv.2010.08.004

Spindler, H. C., \& Norford, L. K. (2009a). Naturally ventilated and mixed-mode buildings-Part I: Thermal modeling. Building and Environment. https://doi.org/10.1016/j.buildenv.2008.05.019

Spindler, H. C., \& Norford, L. K. (2009b). Naturally ventilated and mixed-mode buildings-Part II: Optimal control. Building and Environment. https://doi.org/10.1016/j.buildenv.2008.05.018

Tindale, A. (1993). Third-order lumped-parameter simulation method. Building Services Engineering Research \& Technology. https://doi.org/10.1177/014362449301400302

US-DOE, \& NREL. (2018). EnergyPlus Weather Data. Retrieved from https://energyplus.net/weather

Yoon, N., \& Malkawi, A. (2017). Predicting the Effectiveness of Wind-Driven Natural Ventilation Strategy For Interactive Building Design. Building Simulation, 2163-2170. https://doi.org/10.26868/25222708.2017.587 